Sugerencia de citación: aimesValdez, M. A., Jacobo-Hernández, C.

A. y Ochoa-Jiménez, S. (2021). Los beneficios de la responsabilidad social empresarial: una revisión literaria. tiempo\&economía, 8(2), 201-217. https://doi.org/10.21789/24222704.1720

Vol. 8 N. $0^{\circ} 2$

Julio - Diciembre del 2021

pp. $201-217$

DOI:

https://doi.org/10.21789/24222704.1720

\title{
Los beneficios de la responsabilidad social empresarial: una revisión literaria
}

\section{The Benefits of Corporate Social Responsibility: A Literature Review}

\author{
Miguel Ángel Jaimes Valdez \\ Universidad Autónoma de Baja California, México \\ https://orcid.org/0000-0002-0758-3259 \\ miguel.jaimes@uabc.edu.mx
}

\section{Carlos Armando Jacobo Hernández Instituto Tecnológico de Sonora, México https://orcid.org/0000-0002-8524-6258 cjacobo@itson.edu.mx}

Sergio Ochoa Jiménez Instituto Tecnológico de Sonora, México https://orcid.org/0000-0003-1848-3760

sergio.ochoa@itson.edu.mx

\section{RESUMEN}

La situación mundial actual, caracterizada por problemáticas de tipo social, ambiental y económico, plantea la necesidad de nuevas formas de 
organización. Existen algunos temas que podrían ayudar al respecto, como la responsabilidad social empresarial. Sin embargo, existe confusión en cuanto a los beneficios que esta aporta. Por ello, se realiza una revisión literaria para proporcionar al lector una introducción sobre este tema. Los hallazgos señalan que la responsabilidad social empresarial tiene una relación positiva con temas relevantes como la reputación corporativa, la intención de compra, el voluntarismo, el compromiso, la sostenibilidad y el desempeño organizacional, entre otros. Por ende, es necesario continuar con el desarrollo teórico y práctico de la responsabilidad social por parte de la academia y el sector empresarial, respectivamente.

Palabras clave: responsabilidad social empresarial, administración de negocios, historia económica.

Códigos JEL: M14, M1.

\section{ABSTRACT}

The current world situation, marked by social, environmental, and economic problems, raises the need for new forms of organization, where topics such as corporate social responsibility could help. However, there is confusion about the benefits of this organizational approach. Therefore, a literature review is carried out to provide the reader with an introduction on this topic. Our results indicate that corporate social responsibility has a positive relationship with relevant issues such as corporate reputation, purchase intention, voluntarism, commitment, sustainability, and organizational performance, among others. Consequently, it is necessary to continue with the theoretical and practical development of social responsibility in the academy and the business sector, respectively.

Keywords: Corporate social responsibility, business administration, economic history.

JEL codes: M14, M1.

\section{INTRODUCCIÓN}

La teoría de Kondratieff describe la manera en que las sociedades se desarrollan a través de olas socioeconómicas que duran de cuarenta a sesenta 
años. De acuerdo con los acontecimientos de la crisis mundial de 2008, en la actualidad se está experimentando el final de la quinta ola - caracterizada por la productividad laboral- y el inicio de una sexta - que se enfoca en la productividad de los recursos naturales-, con nuevos detonantes para el desarrollo social, tecnológico y económico que buscan dar solución al cambio climático, previniendo así la destrucción de los ecosistemas (Kurki \&Wilenius, 2014).

Por lo anterior, la responsabilidad social adquiere gran importancia debido a que permite crear nuevas formas de organización que se adhieran a los propósitos descritos en el párrafo anterior. Sin embargo, el concepto de responsabilidad social empresarial (RSE) es identificado de diversas maneras alrededor del mundo, a pesar de haber sido redactado en numerosas normas y recomendaciones. Esto indica que todavía persiste cierta confusión en cuanto a dicho concepto (Freeman \& Hasnaoui, 2011). Por ello, a través de una revisión teórica, se brindará una descripción del origen de la responsabilidad social, su significado y los beneficios que esta genera en las organizaciones.

\section{MARCO TEÓRICO}

El origen de la literatura sobre responsabilidad social se ha identificado en diversas épocas. Mientras que Mareen en 2008 señala la década de los cincuenta, Hoffman en 2007 lo atribuye a los años veinte; Asongu en 2007 se remonta incluso hasta el año 1.700 a. C., en la antigua Mesopotamia. No obstante, tomando en cuenta el tema de la gestión, Frederick Taylor, padre de la administración científica, promueve en su cuarto principio la idea de la cooperación y la responsabilidad de la gerencia hacia sus empleados. Aunado a ello, los magnates de la segunda revolución industrial contribuyeron de manera importante en el mismo sentido. Tal es el caso de John D. Rockefeller, quien señaló que el bienestar del trabajador es tan valioso como la misma producción de bienes y servicios. Por otra parte, Henry Ford destacó al servicio que estaba por encima de las ganancias, a través de la producción con el mínimo costo y la mayor eficiencia, además de puntualizar que la avaricia y el desperdicio son los antagonistas del servicio (Evans et al., 2013).

El inicio de la era moderna de la responsabilidad social se dio en 1953 con la publicación del libro "Social Responsibilities of the Businessman", de 
Howard Bowen, en donde se define a la RSE como la obligación de los empresarios de acatar aquellas regulaciones necesarias para tomar decisiones o seguir líneas de acción que son deseables en término de los objetivos y valores de la sociedad (Carroll, 1999, citado en Murmura et al., 2017). Específicamente, la responsabilidad social en el mundo de los negocios considera las exigencias de la sociedad hacia las organizaciones en el ámbito económico, legal y ético (Carroll, 1979, citado en Chiang et al., 2017).

La RSE se encarga de la relación entre la empresa y la sociedad, la cual puede ser conceptualizada a través de cuatro elementos: la dimensión económica, con el objetivo de lograr ganancias sin comprometer los intereses de los grupos de interés; la dimensión legal, que se refiere a mantener utilidades suficientes mientras que se respetan las diversas leyes y regulaciones gubernamentales; la dimensión ética, al evitar acciones que podrían ser dignas de rechazo por parte de la sociedad; y la dimensión de responsabilidades filantrópicas, al responder a las demandas sociales voluntariamente y con la discreción corporativa necesaria (Bekele et al., 2012). Por su parte, el organismo certificador a escala nacional, denominado Centro Mexicano para la Filantropía (CEMEFI A.C.), considera a la RSE como una nueva forma de gestión y de hacer negocios, a través de la cual una empresa se ocupa de que sus operaciones sean sustentables en lo económico, lo social y lo ambiental (CEMEFI A.C., 2015).

La idea general de la RSE consiste en que las corporaciones deben considerar el impacto de sus actividades no solamente sobre sus accionistas, sino también sobre sus grupos de interés, que son personas, grupos e instituciones (Ott, 2009). A pesar de que la RSE abarca un amplio rango de actividades, como la fabricación de productos con materiales biodegradables, el desempeño de un trabajo cercano a las comunidades o la donación a fundaciones que realizan actividades de caridad (McCarthy et al., 2017), una forma simple de identificar la RSE son las acciones que benefician (parcial o completamente) a la sociedad y que no son obligatorias por ley (Arvidsson, 2010, citado en Jong \& Meer, 2017). La importancia del asunto surge cuando las empresas que adoptan los códigos de buenas prácticas de RSE son reconocidas globalmente como aquellas que se esfuerzan por minimizar los efectos adversos de sus operaciones en la comunidad y el medioambiente (Lim \& Phillips, 2008, citado en Darus et al., 2017). Es necesario destacar que mientras las exigencias sociales provienen de los empleados y de las organizaciones no gubernamentales (ONG), las demandas de tipo ambiental 
surgen de los clientes, las instituciones crediticias y el gobierno, en general (Meixell \& Luoma, 2015, citado en Haleem et al., 2017).

La RSE se ha convertido en una parte integral de la administración de los negocios en el mundo, ya que sus representantes se encargan de erogar gastos sustanciales en actividades de esta naturaleza. De acuerdo con el reporte de Inversiones Responsables y Sostenibles (SRI), las inversiones en este tipo de rubros incrementaron 76 \% en el período 2012-2014 (Hasan \& Habib, 2017). Adicionalmente, $80 \%$ de las empresas comunican sus actividades de RSE a través de sus portales de internet (PricewaterhouseCooper, 2010, citado en Byun \& Oh, 2017). Esto no debe extrañar, puesto que la RSE contribuye a la disminución de costos, además de estar asociada con la calidad y una mejor reputación de la empresa (Maignan et al., 1999, citado en Charlo-Molina \& Moya-Clemente, 2010).

\section{METODOLOGÍA}

El presente trabajo es producto de una revisión teórica con un diseño exploratorio, la cual contempla el análisis de artículos científicos relacionados con la RSE. Siguiendo a Creswell (2009), las cuatro etapas implementadas fueron: (i) seleccionar la base de datos científica de Elsevier; (ii) las palabras clave utilizadas para la descarga de los artículos empíricos publicados en el período 2015-2017 fueron "Corporate Social Responsibility"; (iii) se llevó a cabo un análisis exhaustivo del país sede, los antecedentes, los asuntos tratados y los resultados; (iv) y se realizó una discusión -es decir, una comparación entre documentos-, identificando contrastes y semejanzas, para finalmente proporcionar las conclusiones.

\section{ANÁLISIS DE RESULTADOS}

El impacto de la RSE en las organizaciones es diverso e incluye numerosos beneficios para las mismas. Entre los temas que destacan están la sostenibilidad, la motivación laboral, la innovación, el desempeño organizacional y la mejora de la imagen pública, entre otros (tabla 1). 


\begin{tabular}{|c|c|c|}
\hline Relación positiva de la RSE con & País & Referencia \\
\hline Compromiso organizacional & Corea del Sur & Kim et al. (2016) \\
\hline Reputación corporativa y satisfacción del cliente & China & Su et al. (2017) \\
\hline Desempeño financiero & España & $\begin{array}{c}\text { García-Madariaga y Rodrí- } \\
\text { guez-Rivera (2017) }\end{array}$ \\
\hline Desempeño financiero & España & Rodriguez-Fernandez (2015) \\
\hline Desempeño competitivo & España & Herrera-Madueño et al. \\
(2015)
\end{tabular}

Tabla 1. Impacto de la RSE en las organizaciones

Fuente: elaboración propia con base en las fuentes de información incluidas.

Un estudio presentado por Kim et al. (2016) intenta demostrar una relación entre la RSE —integrada por las dimensiones económica, legal, ética y de responsabilidad filantrópica - y el compromiso organizacional - que trata sobre la dedicación, la lealtad y la creencia en el valor de la corporación por parte de los empleados - compuesta por el bienestar laboral, la capacitación, la compensación, la comunicación y el apoyo administrativo. Para tales efectos, se diseñó un cuestionario que fue aplicado a 310 empleados de uno de los casinos más importantes de Corea del Sur. Los resultados, obtenidos a través del método de modelo de ecuaciones estructurales, indican una relación positiva entre ambas variables, es decir, el compromiso organizacional de los trabajadores puede mejorarse con los programas de RSE. Además, estos últimos tienen un efecto en la disminución del ausentismo laboral, lo que mejora la eficiencia de la empresa.

Otro estudio realizado en Asia lo celebran Su et al. (2017) para investigar el impacto de la RSE y las actitudes de los clientes; específicamente en cuanto a la reputación corporativa (juicio colectivo sobre una empresa que predice futuras intenciones de compra) y la satisfacción del producto (asociación emocional positiva hacia la empresa que es vital para una relación 
sostenible a largo plazo). Con ese objetivo, se aplicó un cuestionario a 451 clientes de tres hoteles en China, el cual arrojó una correlación positiva entre ambas variables con ayuda del método de modelo de ecuaciones estructurales. Por lo anterior, las actividades de RSE ayudan a construir una relación más fuerte entre la empresa de servicios y sus huéspedes al mejorar la satisfacción del cliente y la reputación corporativa.

En España, se trata de dar respuesta a la influencia de la RSE sobre el valor de mercado de las organizaciones del sector automotriz. Para ello, se tomaron como base los datos del desempeño en RSE de las empresas de este sector durante ocho años, los cuales señalan que, efectivamente, la RSE puede tener una relación con un mejor desempeño financiero (GarcíaMadariaga \& Rodríguez-Rivera, 2017). En ese mismo país se desarrolla una investigación similar que busca determinar la relación entre la RSE y el desempeño financiero de las empresas enlistadas en la Bolsa de Madrid mediante el análisis de índices como Global Reporting Initiative participation, Dow Jones Sustainability Index firm inclusion, Good Corporate Governance Recommendations compliance y Global Compact signee. Los resultados indican que todas las políticas sociales incrementan los recursos financieros y viceversa; es decir, el éxito financiero conlleva a mayores beneficios sociales (Rodriguez-Fernandez, 2015).

Otro estudio pretende demostrar la relación entre la RSE y el desempeño competitivo de las pequeñas y medianas empresas en España mediante una muestra de 481 sujetos y el método estadístico de ecuaciones estructurales. Una vez finalizado, se encuentra que existe una relación directa e indirecta entre ambos conceptos, particularmente a través de la gestión de los grupos de interés (Herrera-Madueño et al., 2015).

Gras-Gil et al. (2016) Ilevan a cabo un estudio para identificar la relación entre la RSE y la gestión de ganancias. El experimento involucró la alteración de los reportes financieros de una empresa para distraer a algunos grupos de interés o influenciar compromisos contractuales con el fin de aprovechar oportunidades en el mercado de capitales, obtener financiamiento externo y cumplir con la reglamentación gubernamental, entre otros. La muestra estuvo compuesta por las 100 empresas con mayor reputación de España (excluyendo a las entidades financieras), según el Índice Merco, para el período 2005-2012. Los resultados señalan que existe una relación negativa entre las actividades de RSE y la gestión de ganancias. Así mismo, se comprobó una 
relación positiva entre la RSE y la satisfacción del cliente, la eficiencia en el uso de los recursos y la satisfacción de los grupos de interés.

En el mismo año, Reverte et al. (2016) estudiaron la relación de la RSE (en sus dimensiones económica, social y ambiental) con la innovación (nuevos productos, servicios, procesos y formas de trabajar que son fuente de una ventaja comparativa) y el desempeño organizacional, el cual toma en cuenta indicadores financieros (crecimiento en las ventas y nivel de productividad, entre otros) y no financieros (calidad de los productos/servicios, satisfacción del cliente, motivación de los empleados, entre otros). Los sujetos de estudio fueron 133 organizaciones españolas —en su mayoría pequeñas y medianas empresas- que manifestaron ser ecológicamente responsables. A través de la aplicación del método de modelo de ecuaciones estructurales, se determinó una relación positiva y significativa de la RSE con la innovación y el desempeño organizacional.

Un estudio a nivel mundial lo proporcionan Cycyota et al. (2016), quienes consultaron los informes anuales de las empresas enlistadas en la clasificación de las 100 mejores para trabajar según la revista Fortune, con el objetivo de identificar el impacto de las acciones que llevan a cabo las organizaciones que aseguran la implementación de la RSE y el voluntarismo de los empleados (la acción de proporcionar energía, habilidades o talentos a una organización de caridad sin recibir pago alguno). El voluntarismo cuenta con beneficios internos, como una mayor motivación y satisfacción de los empleados, contribuyendo además al desarrollo de habilidades. En el ámbito externo, se mejora la reputación, las utilidades y legitimidad de la empresa.

En el Medio Oriente, Mehralian et al. (2016) analizaron la relación entre la RSE y el desempeño organizacional (las cuatro dimensiones del cuadro de mando integral: finanzas, clientes, procesos internos y aprendizaje) a través de la aplicación del método de modelo de ecuaciones estructurales sobre una muestra de 933 cuestionarios enviados a empresas farmacéuticas en Irán. Los resultados indican que la RSE puede influir indirectamente en el desempeño de la empresa, además de mejorar las prácticas del sistema de gestión de calidad.

Una nueva investigación en la misma región fue desarrollada por Asrar-ul-Haq et al. (2017) con la finalidad de encontrar la relación entre la RSE y las variables de compromiso organizacional (afiliación e involucramiento de un empleado con su organización) y satisfacción laboral (estado 
emocional positivo como resultado de la experiencia de trabajo). El objeto de estudio estuvo representado por 245 empleados universitarios de 14 campus y 8 universidades en Pakistán. A través de la aplicación del método de modelo de ecuaciones estructurales, se determinó una relación significativa entre los temas antes descritos.

En otra parte del mundo, Akdogan et al. (2016) desarrollaron un estudio sobre la relación entre RSE y la identificación organizacional. Esta investigación trata sobre un sentido fundamental de identidad que refleja un estado psicológico donde se comparten atributos entre la organización y la persona. Este trabajo aplicó un cuestionario a 323 empleados de una empresa de aviación en Turquía que demostró una relación positiva entre ambas variables. En el mismo país, Uzunoglu et al. (2017) identificaron una relación positiva entre la RSE y la intención de compra de los clientes a través de un experimento de redes sociales en el que participaron 253 personas. Lo anterior indica que una RSE administrada y comunicada satisfactoriamente frente a sus clientes es una herramienta esencial en el trato de los grupos de interés por parte de las organizaciones.

\section{DISCUSIÓN}

Los resultados indican que la RSE tiene una relación positiva con el compromiso organizacional (Asrar-ul-Haq et al., 2017; Kim et al., 2016) que trata sobre la dedicación, la lealtad y la creencia en el valor de la corporación por parte de los empleados. Esto corresponde al compromiso señalado por Bostrom et al. (2014), el cual privilegia el diálogo, la responsabilidad y el aprendizaje.

Algunos estudios cuantitativos demuestran una relación positiva entre la RSE y el desempeño financiero en España, tanto en el sector automotriz (García-Madariaga \& Rodríguez-Rivera, 2017) como en las empresas enlistadas en los índices bursátiles más importantes del mundo referentes a la RSE y la sostenibilidad (Rodriguez-Fernandez, 2015). Esto concuerda con investigaciones que abordan precisamente la relación entre la RSE y el desempeño sostenible (Herbohn et al., 2014), aunque en el sector minero y energético de Australia.

El desempeño competitivo también posee una relación con la RSE, en especial con la gestión de los grupos de interés (Herrera-Madueño 
et al., 2015), lo cual señala que es sumamente importante mantener una comunicación directa con socios, empleados, proveedores, clientes y demás participantes. Por lo anterior, no es de extrañar que la RSE tenga una relación con el liderazgo (Spitzeck, 2009), reflejada en la creación de un mayor número de comités de RSE en las empresas; sobre todo para lograr un lugar en el Corporate Responsibility Index.

La RSE guarda una relación con la reputación corporativa (Gras-Gil et al., 2016; Su et al., 2017) y la intención de compra (Uzunoglu et al., 2017), entendida como el juicio de valor que ejecutan los clientes respecto a la compañía y que influye en las ventas de la misma. Esto es importante, ya que a la reputación empresarial se le ha vinculado también con la reducción de costos y la mejora de resultados en las finanzas corporativas (Dadhich et al., 2015) y parte del valor de los activos intangibles de la organización (McWilliams \& Siegel, 2000, citado en Wagner, 2010).

Por otra parte, la RSE se relaciona también con el desempeño organizacional (Mehralian et al., 2016; Reverte et al., 2016), tomando en cuenta el cuadro de mando integral y los indicadores financieros y no financieros. Aunado a ello, se ha comprobado que existe una correlación significativa entre el desempeño organizacional (fuente de ventaja competitiva) y el clima laboral para la innovación (Shanker et al., 2017).

Finalmente, la RSE mantiene una relación positiva con la identificación organizacional (Akdogan et al., 2016), lo cual es consistente con el mismo tipo de relación encontrada por Glavas y Godwin (2013, citado en Matherne et al., 2017). Así mismo, se evidencia la relación de la RSE con el voluntarismo de los empleados (Cycyota et al., 2016), algo señalado hace cinco décadas por Walton (1967, citado por Freeman \& Hasnoui, 2011), específicamente la necesidad del voluntarismo y su integración en la RSE.

En suma, los artículos científicos descritos anteriormente han aportado evidencia relevante acerca de los beneficios de la RSE a través de la relación que esta guarda con otras variables. Por lo anterior, se contribuye a la idea que califica a la RSE como el punto fundamental que influye en el desempeño de la empresa (Laguir et al., 2015). Esto contrasta con lo mencionado en un artículo del New York Times escrito hace aproximadamente cincuenta años, que se pronuncia acerca de la RSE como un instrumento que solamente trata de aumentar las ganancias de las empresas (Friedman, 1970, citado en Cronqvist \& Yu, 2017). En realidad, la RSE, más que un medio exclusivo como paragón de utilidades, es una parte integral de la organización que 
se asemeja a la tercera era del gobierno corporativo denominada grupos de interés, la cual fue propuesta por Freeman en 1984 y que se pronuncia por el equilibrio en el logro de los objetivos de la empresa y los grupos de interés (Mostovicz et al., 2011). Así, la empresa no solamente es una entidad con ánimo de lucro que privilegia la dimensión económica, sino que va más allá de tal propósito, involucrando otras dimensiones, como la social y la ambiental, lo cual se ha comprobado previamente con la relación positiva de la RSE con la sostenibilidad (Sharma \& Khanna, 2014).

Algo que se ha detectado es que la mayoría de las investigaciones sobre RSE tratan sobre la comprobación de correlaciones a través del método cuantitativo. Por ende, se podría complementar el análisis con un mayor número de investigaciones cualitativas para conocer mejor a los grupos de interés. Un ejemplo de esto es que la literatura sobre RSE está llena de buenas prácticas, pero no existe una guía de cómo las empresas deben crear sus propias políticas de RSE, y mucho menos cómo implementarlas (Bhattacharya \& Sen, 2004, citado en Lindgreen et al., 2009). Tal vez, la clave para el desarrollo de la RSE en los próximos años se encuentre en recordar la importancia de la solidaridad entre las organizaciones y la sociedad, donde el valor compartido sea considerado en la toma de decisiones de los empresarios para el beneficio mutuo, como han propuesto Michelini y Fiorentino (2012).

\section{CONCLUSIONES}

En el presente escrito se ha proporcionado una descripción del origen, desarrollo e impacto de la RSE en las organizaciones, demostrando que no se trata únicamente de un tema fugaz, sino que algunos rasgos teóricos se remontan a la antigüedad. Además, se señala que existen diversos autores que han aportado al desarrollo teórico del tema y que es menester consultarlos para enriquecer la discusión filosófica de la responsabilidad empresarial. Adicionalmente, se han comprobado numerosos beneficios de la RSE, sobre todo a través de estudios cuantitativos alrededor del mundo, entre los cuales destacan la eficiencia, la innovación, la reputación corporativa, la sostenibilidad y la satisfacción del cliente.

Por lo anterior, la RSE no debe reducirse a un simple distintivo con el único fin de mejorar la imagen de las organizaciones, sino que debe ser un tema digno de reconocimiento, consultando sus beneficios y aplicándolos 
al mayor número de empresas alrededor del mundo, sin perder de vista el debate entre la teoría y la práctica. Así mismo, la unificación de criterios para clasificar a una empresa con el distintivo de RSE es un tema indispensable. Esto podría plantearse no solamente desde la perspectiva teórica, que evidentemente es la fundamental, sino que también habría que acotar el tiempo y el espacio en que opera la misma, además de identificar fehacientemente a sus grupos de interés, generar un espacio de diálogo y lograr acuerdos que beneficien a todas las partes. Todo esto sería de gran ayuda para la conservación de la paz y la tranquilidad, y por consiguiente la supervivencia del ser humano.

\section{REFERENCIAS}

Akdogan, A., Arslan, A., \& Demirtas, O. (2016). A strategic influence of corporate social responsibility on meaningful work and organizational identification, via perceptions of ethical leadership. Procedia - Social and Behavioral Sciences, 235, 259-268. http://dx.doi. org/10.1016/j.sbspro.2016.11.029

Asrar-ul-Haq, M., Kuchinke, K., \& Iqbal, A. (2017). The relationship between corporate social responsibility, job satisfaction, and organizational commitment: Case of Pakistani higher education. Journal of Cleaner Production, 142, 2352-2363. http://dx.doi.org/10.1016/j. jclepro.2016.11.040

Bekele, A., Bosona, T., Nordmark, I., Gebresenbet, G., \& Ljungberg, D. (2012). Assessing the sustainability of food retail business: The case of Konsum Värmland, Sweden. Journal of Service Science and Management, 5, 373-385. http://dx.doi.org/10.4236/jssm.2012.54044

Bostrom, M., Jonsson, A. M., Lockie, S., Mol, A. P. J., \& Oosterveer, P. (2014). Sustainable and responsible supply chain governance: Challenges and opportunities. Journal of Cleaner Production, 107, 1-7. http:// dx.doi.org/10.1016/j.jclepro.2014.11.050

Byun, S., \& Oh, S. (2017). Local corporate social responsibility, media coverage, and shareholder value. Journal of Banking and Finance, 87, 68-86. http://dx.doi.org/10.1016/j.jbankfin.2017.09.01 
Centro Mexicano para la Filantropía [CEMEFI A.C.]. (2015). El concepto de responsabilidad social empresarial. http://www.cemefi.org/esr/ images/stories/pdf/esr/concepto_esr.pdf

Charlo-Molina, M. J., \& Moya-Clemente, I. (2010). El comportamiento financiero de las empresas socialmente responsables. Investigaciones Europeas de Dirección y Economía de la Empresa, 16(2), 15-25. http:// www.redalyc.org/articulo.oa?id $=274120099001$

Chiang, W., Shang, J., \& Sun, L. (2017). Broad bond rating change and irresponsible corporate social responsibility activities. Advances in Accounting, 39, 32-46. http://dx.doi.org/10.1016/j.adiac.2017.09.002

Creswell, J. W. (2009). Research design: Qualitative, quantitative, and mixed methods approaches. SAGE.

Cronqvist, H., \& Yu, F. (2017). Shaped by their daughters: Executives, female socialization, and corporate social responsibility. Journal of Financial Economics, 126(3), 543-562. https://doi.org/10.1016/j. jfineco.2017.09.00

Cycyota, C., Ferrante, C., \& Schoeder, J. M. (2016). Corporate social responsibility and employee volunteerism: What do the best companies do? Business Horizons, 59, 321-329. http://dx.doi. org/10.1016/j.bushor.2016.01.004

Dadhich, P., Genovese, A., Kumar, N., \& Acquaye, A. (2015). Developing sustainable supply chains in the UK construction industry: A case study. Management of Environmental Quality: An International Journal, 25(4), 431-445. http://dx.doi.org/10.1016/j.ijpe.2014.12.012

Darus, F., Ahmad-Shukri, A., Yusoff, H., Ramlia, A., Zain, M., \& Abu-Bakar, N. (2017). Empowering social responsibility of Islamic organizations through Waqf. Research in International Business and Finance, 42, 959965. http://dx.doi.org/10.1016/j.ribaf.2017.07.030

Evans, R., Pane-Haden, S., Clayton, R., \& Novicevic, M. (2013). History-of-management-thought about social responsibility. Journal of Management History, 19(1), 8-32. http://dx.doi. org/10.1108/17511341311286150

Freeman, I., \& Hasnaoui, A. (2011). The meaning of corporate social responsibility: The vision of four nations. Journal of Business Ethics, 100, 419-443. http://dx.doi.org/10.1007/s10551-010-0688-6 
García-Madariaga, J., \& Rodríguez-Rivera, F. (2017). Corporate social responsibility, customer satisfaction, corporate reputation, and firms' market value: Evidence from the automobile industry. Spanish Journal of Marketing, 21(S1), 39-53. http://dx.doi.org/10.1016/j. sjme.2017.05.003

Gras-Gil, E., Palacios-Manzano, M., \& Hernández-Fernández, J. (2016). Investigating the relationship between corporate social responsibility and earnings management: Evidence from Spain. $B R Q$ Business Research Quarterly, 19, 289-299. http://dx.doi.org/10.1016/j. brq.2016.02.002

Haleem, F., Farooq, S., \& Vejrum-Wæhrens, B. (2017). Supplier corporate social responsibility practices and sourcing geography. Journal of Cleaner Production, 153, 92-103. http://dx.doi.org/10.1016/j. jclepro.2017.03.143

Hasan, M., \& Habib, A. (2017). Corporate life cycle, organizational financial resources and corporate social responsibility. Journal of Contemporary Accounting \& Economics, 13, 20-36. http://dx.doi. org/10.1016/j.jcae.2017.01.002

Herbohn, K., Walker, J., \& Yien-Loo, H. M. (2014). Corporate social responsibility: The link between sustainability disclosure and sustainability performance. ABACUS, 50(4), 422-459. http://dx.doi. org/10.1111/abac.12036

Herrera-Madueño, J., Larrán-Jorge, M., Martínez-Conesa, I., \& MartínezMartínez, D. (2015). Relationship between corporate social responsibility and competitive performance in Spanish SMEs: Empirical evidence from a stakeholders' perspective. BRQ Business Research Quarterly, 19(1), 55-72. http://dx.doi.org/10.1016/j. brq.2015.06.002

Jong, M., \& Meer, M. (2017). How does it fit? Exploring the congruence between organizations and their corporate social responsibility (CSR) activities. Journal of Business Ethics, 143, 71-83. http://dx.doi. org/10.1007/s10551-015-2782-2

Kim, J., Jun-Song, H., \& Lee, C. (2016). Effects of corporate social responsibility and internal marketing on organizational commitment and turnover intentions. International Journal of Hospitality Management, 55, 25-32. http://dx.doi.org/10.1016/j.ijhm.2016.02.007 
Kurki, S., \& Wilenius, M. (2014). Organisations and the sixth wave: Are ethics transforming our economies in the coming decades? Futures, 71, 146-158. http://dx.doi.org/10.1016/j.futures.2014.09.001

Laguir, I., Stagliano, R., \& Elbaz, J. (2015). Does corporate social responsibility affect corporate tax aggressiveness? Journal of Cleaner Production, 107, 662-675. http://dx.doi.org/10.1016/j. jclepro.2015.05.059

Lindgreen, A., Swaen, V., \& Johnston, W. (2009). Corporate social responsibility: An empirical investigation of U.S. organizations. Journal of Business Ethics, 85, 303-323. http://dx.doi.org/10.1007/ s10551-008-9738-8

Matherne, C., Waterwall, B., Ringb, J. K., \& Credo, K. (2017). Beyond organizational identification: The legitimization and robustness of family identification in the family firm. Journal of Family Business Strategy, 8, 170-184. http://dx.doi.org/10.1016/j.jfbs.2017.08.001

McCarthy, S., Oliver, B., \& Song, S. (2017). Corporate social responsibility and CEO confidence. Journal of Banking and Finance, 75, 280-291. http://dx.doi.org/10.1016/j.jbankfin.2016.11.02

Mehralian, G., Nazari, J., Zarei, L., \& Rasekh, H. (2016). The effects of corporate social responsibility on organizational performance in the Iranian pharmaceutical industry: The mediating role of TQM. Journal of Cleaner Production, 135, 689-698. http://dx.doi.org/10.1016/j. jclepro.2016.06.116

Michelini, L., \& Fiorentino, D. (2012). New business models for creating shared value. Social Responsibility Journal, 8(4), 561-577. http:// dx.doi.org/10.1108/17471111211272129

Mostovicz, E. I., Kakabadse, N. K., \& Kakabadse, A. (2011). Corporate governance: quo vadis? Corporate Governance: The International Journal of Business in Society, 11(5), 613-626. http://dx.doi. org/10.1108/14720701111177019

Murmura, F., Bravi, L., \& Palazzi, F. (2017). Evaluating companies' commitment to corporate social responsibility: Perceptions of the SA 8000 standard. Journal of Cleaner Production, 164, 1406-1418. http:// dx.doi.org/10.1016/j.jclepro.2017.07.073 
Ott, C. (2009). Corporate governance and corporate social responsibility in profit- and in non-profit-organization. Journal fur Rechtspolitik, 17, 255-262. http://dx.doi.org/10.1007/s00730-009-0286-z

Reverte, C., Gomez-Melero, E., \& Cegarra-Navarro, J. (2016). The influence of corporate social responsibility practices on organizational performance: Evidence from eco-responsible Spanish firm. Journal of Cleaner Production, 112, 2870-2884. http://dx.doi.org/10.1016/j. jclepro.2015.09.128

Rodriguez-Fernandez, M. (2015). Social responsibility and financial performance: The role of good corporate governance. BRQ Business Research Quarterly, 19(2), 137-151. http://dx.doi.org/10.1016/j. brq.2015.08.001

Shanker, R., Bhanugopan, R., van-der-Heijden, B. I., \& Farrell, M. (2017). Organizational climate for innovation and organizational performance: The mediating effect of innovative work behavior. Journal of Vocational Behavior, 100, 67-77. http://dx.doi.org/10.1016/j. jvb.2017.02.004

Sharma, J. P. (2014). Corporate social responsibility, corporate governance and sustainability: Synergies and inter-relationships. Indian Journal of Corporate Governance, 7(1), 14-38. http://dx.doi. org/10.1177/0974686220140102

Spitzeck, H. (2009). The development of governance structures for corporate responsibility. Corporate Governance: The international journal of business in society, 9(4), 495-505. http://dx.doi. org/10.1108/14720700910985034

Su, L., Pan, Y., \& Chen, X. (2017). Corporate social responsibility: Findings from the Chinese hospitality industry. Journal of Retailing and Consumer Services, 34, 240-247. http://dx.doi.org/10.1016/j. jretconser.2016.10.013

Uzunoglu, E., Turkel, S., \& Yaman-Akyar, B. (2017). Engaging consumers through corporate social responsibility messages on social media: An experimental study. Public Relations Review, 43(5), 989-997. http:// dx.doi.org/10.1016/j.pubrev.2017.03.013 
Wagner, M. (2010). The role of corporate sustainability performance for economic performance: A firm-level analysis of moderation effects. Ecological Economics, 69(7), 1553-1560. http://dx.doi.org/10.1016/j. ecolecon.2010.02.017 